\title{
Article \\ The Speed of Convergence of the Threshold Estimator of Ruin Probability under the Tempered $\alpha$-Stable Lévy Subordinator
}

\author{
Yuan Gao ${ }^{1}$ and Honglong You ${ }^{2, *}$ \\ 1 School of Mathematical Sciences, Qufu Normal University, Jining 273165, China; gyyq1413@163.com \\ 2 School of Statistics, Qufu Normal University, Jining 273165, China \\ * Correspondence: yougaoyou815@qfnu.edu.cn
}

\begin{abstract}
In this paper, a nonparametric estimator of ruin probability is introduced in a spectrally negative Lévy process where the jump component is a tempered $\alpha$-stable subordinator. Given a discrete record of high-frequency data, a threshold technique is proposed to estimate the mean of the jump size and use the Fourier transform and the Pollaczek-Khinchin formula to construct the estimator of ruin probability. The convergence rate of the integrated squared error for the estimator is studied.
\end{abstract}

Keywords: ruin probability; spectrally negative Lévy process; Fourier transform; high-frequency data

MSC: 62G20; 62M05

check for updates

Citation: Gao, Y.; You, H. The Speed of Convergence of the Threshold Estimator of Ruin Probability under the Tempered $\alpha$-Stable Lévy Subordinator. Mathematics 2021, 9, 2654. https://doi.org/10.3390/ math9212654

Academic Editor: Mark Kelbert

Received: 14 September 2021

Accepted: 18 October 2021

Published: 20 October 2021

Publisher's Note: MDPI stays neutral with regard to jurisdictional claims in published maps and institutional affiliations.

Copyright: (c) 2021 by the authors. Licensee MDPI, Basel, Switzerland. This article is an open access article distributed under the terms and conditions of the Creative Commons Attribution (CC BY) license (https:/ / creativecommons.org/licenses/by/ $4.0 /)$.

\section{Introduction}

In actuarial science, the statistical inference of ruin probability has received much attention from scholars. Many works have been contributed to parametric and nonparametric estimation of ruin probability. See, for example, Croux and Veraverbeke [1], Frees [2], Mnatsakanov et al. [3], Pitts [4], Politis [5], and Veraverbeke [6]. In recent years, many nice results have been obtained by actuarial scholars, such as Huang et al. [7], Li et al. [8], You et al. [9], Zhang and Yang [10], Zhang and Yang [11], Zhang [12], and Zhang and Yang [13]. As an extension of ruin probability, the Gerber-Shiu function has been introduced and studied for its statistical properties. Interested readers can refer to Su and $\mathrm{Yu}$ [14,15], Yang et al. [16], Zhang and Su [17], Su et al. [18], Zhang and Su [19], Zhang [20], Shimizu [21], and Shimizu and Zhang [22], among others.

In Asmussen and Albrecher [23], an analytic (or probabilistic) approach was suggested, and it needs much more detailed information about the risk model, such as the claim size distribution. However, in practical situations, it is not easy to obtain the specific distribution information. Instead, one observes the surplus process at some discrete time points. Then, a statistical methodology can be directly used to estimate the claim size distribution with the observed data. In Zhang and Yang [10], a nonparametric estimator of ruin probability was proposed, based on the Pollaczek-Khinchin formula and the Fourier transform in a pure-jump Lévy risk model. This estimation approach was extended by Zhang and Yang [11] to a spectrally negative Lévy risk model. Subsequently, Shimizu and Zhang [22] estimated the Gerber-Shiu function for an insurance surplus process driven by a Lévy subordinator. In Zhang and Yang [10] and Comte and Genon-Catalot [24], they considered high-frequency sampling with $n$ discrete time observations of step width $h_{n}>0$ and derived asymptotics under the framework that $h_{n} \rightarrow 0$ and $n h_{n} \rightarrow \infty$.

In the present work, our interest is to estimate ruin probability for a spectrally negative Lévy risk model under the above framework. Assume that the surplus of the risk model can be observed at a sequence of discrete time points $\left\{t_{k}^{n}=k h_{n}, k=0,1,2,3, \ldots\right\}$ with $h_{n} \geq 0$ being the length of the sampling interval. Without observing the jump and diffusion 
parts of the risk model, it is challenging to estimate ruin probability, since it depends on both parts in a spectrally negative Lévy risk model. In Mancini [25-28] and Shimizu [29,30], they developed a threshold technique for identifying the times when jump sizes exceed a suitably defined threshold. Using the threshold technique and the Fourier transform, an estimator of ruin probability is constructed, and the convergence rate of its integrated squared error is obtained.

The remainder of this paper is organized as follows. In Section 2, the risk model, as well as some assumptions for the asymptotic theory are introduced. In Section 3, some estimators are suggested, based on the Fourier transform and the threshold technique. In Section 4, the convergence rate of our estimators is established. In Section 5, we conclude this paper.

\section{Preliminaries}

\subsection{Risk Model and Some Assumptions}

A spectrally negative Lévy process is specified by:

$$
Y_{t}=c t+\sigma W_{t}-J_{t}, \quad t \geq 0,
$$

where $c>0$ is a parameter, $\sigma>0$ represents the perturbation coefficient, $W_{t}$ is a standard Brownian motion, and $J_{t}$ is a subordinator. Suppose that $W_{t}$ and $J_{t}$ are independent of each other. Then, the characteristic exponent of $Y_{t}$ is given by:

$$
\psi_{Y}(s)=\frac{1}{t} \ln \left(\mathbf{E}\left[e^{i s Y_{t}}\right]\right)=i c s-\frac{1}{2} \sigma^{2} s^{2}-\int_{0}^{\infty}\left(1-e^{-i s x}\right) v(d x),
$$

where $v$ is the Lévy measure on $(0, \infty)$. By Sato [31], it can be rewritten as:

$$
J_{t}=L_{t}+M_{t}^{*}
$$

where $L_{t}$ is the sum of jumps over $[0, t]$ with the jump size larger than one, and $M_{t}^{*}$ is the sum of jumps over $[0, t]$ with the jump size less than one. Specifically, $L_{t}=$ $\int_{0}^{t} \int_{x>1} x \mu(d s, d x)=\sum_{k=1}^{N_{t}} \gamma_{k}$, where $\mu$ is the Poisson random measure of $J_{t}$ such that $E[\mu(d s, d x)]=v(d x) d s, N_{t}$ is a Poisson process, and $\gamma_{1}, \gamma_{2}, \gamma_{3}, \ldots$ are i.i.d. random variables, that is $L_{t}$ is a compound Poisson process representing the jumps of $J_{t}$ with the jump size larger than one. Process $M_{t}^{*}$ admits decomposition, $M_{t}^{*}=b t+M_{t}$, where $b=\int_{x \leq 1} x v(d x)$ and $M_{t}=\int_{0}^{t} \int_{x \leq 1} x \tilde{\mu}(d s, d x)$ is a martingale with $\tilde{\mu}(d s, d x)=\mu(d s, d x)-v(d x) d s$ being the compensated measure of $\mu(d s, d x)$. It is known that $M_{t}$ is a square integrable martingale with infinite activity of the jump such that $E\left[M_{t}\right]=0$ and $\operatorname{Var}\left[M_{t}\right]=t \int_{x \leq 1} x^{2} v(d x)<\infty$. Suppose that $\gamma_{k}, N_{t}$, and $M_{t}$ are independent of each other.

Let $u>0$ be the initial surplus of an insurance company. Then, the surplus at time $t$ can be modeled by:

$$
U_{t}=u+Y_{t}=u+c t+\sigma W_{t}-J_{t}, \quad t \geq 0,
$$

where $c$ is the rate of the premium, $\sigma$ represents the perturbation coefficient, $J_{t}$ denotes the claim payments and other expenses in insurance businesses, and $W_{t}$ is a perturbation.

\subsection{Ruin Probability and Its Fourier Transform}

The infinite-time horizon ruin probability $\Phi(u)$ is defined as:

$$
\Phi(u)=\mathbf{P}\left(\inf _{0 \leq t<\infty} U_{t} \leq 0 \mid U_{0}=u\right)
$$

By Equation (1) in Zhang and Yang [11], $\Phi(u)$ admits the following representation:

$$
\Phi(u)=1-(1-\rho) \sum_{i=0}^{\infty} \rho^{i}\left(G^{(i+1) *} * H^{(i) *}\right)(u), \quad u>0,
$$


where $\rho=\frac{\mu_{1}}{c}, \mu_{1}=\int_{0}^{\infty} x v(d x), H(x)=\frac{1}{\mu_{1}} \int_{0}^{x} v(y, \infty) d y$, and $G$ is determined by the Fourier transform $\int_{0}^{\infty} e^{i s x} d G(x)=c\left\{c-\frac{\sigma^{2}}{2} i s\right\}^{-1}$. Setting $\Phi(u) \equiv 0$ for $u<0$, Zhang and Yang [11] obtained the Fourier transform of $\Phi(u)$ :

$$
\begin{aligned}
\mathcal{F}_{\Phi}(s)=\int_{0}^{\infty} e^{i s u} \Phi(u) d u & =\frac{\frac{\sigma^{2}}{2} i s+\frac{1}{i s} \int_{0}^{\infty}\left(e^{i s x}-1\right) v(d x)-\mu_{1}}{i c s+\frac{\sigma^{2}}{2} s^{2}-\int_{0}^{\infty}\left(e^{i s x}-1\right) v(d x)} \\
& =\frac{i c s(1-\rho)+\psi_{Y}(-s)}{-i s \psi_{Y}(-s)}
\end{aligned}
$$

Once an estimator of $\mathcal{F}_{\Phi}(s)$ is available, $\Phi(u)$ can be estimated by the inverse Fourier transform.

\section{Estimation of Ruin Probability}

Suppose that a discrete sample $Y^{n}=\left\{Y_{t_{i}^{n}} \mid t_{i}^{n}=i h_{n} ; i=0,1,2 \ldots, n\right\}$ can be observed. Let $Z_{i}=Y_{t_{i}^{n}}-Y_{t_{i-1}^{n}}, h_{n}=t_{i}^{n}-t_{i-1}^{n}>0, \lim _{n \rightarrow \infty} h_{n}=0$, and $\lim _{n \rightarrow \infty} n h_{n}=\infty$. Our interest is to estimate $\Phi(u)$ by $Z_{1}, Z_{2}, \ldots, Z_{n}$ when Lévy measure $v$ and perturbation coefficient $\sigma$ are unknown.

If one can estimate $\rho$ and $\psi_{Y}(-s)$ in (6), then $\mathcal{F}_{\Phi}(s)$ can be estimated with the plug-in device. Inspired by Zhang and Yang [10,11] and You and Yin [32], we define the estimator of $\psi_{Y}(s)$ :

$$
\hat{\psi}_{Y}(s)=\frac{1}{h_{n}}\left(\frac{1}{n} \sum_{k=1}^{n} e^{i s Z_{k}}-1\right) .
$$

To estimate $\rho=\frac{\mu_{1}}{c}$, we need to estimate $\mu_{1}$, the mean of $J_{1}$. Zhang and Yang [11] proposed to estimate $\mu_{1}$, by:

$$
\hat{\mu}_{1}^{*}=\frac{1}{n h_{n}} \sum_{k=1}^{n}\left(c h_{n}-Z_{k}\right)
$$

Note that $c h_{n}-Z_{k}=\left(J_{t_{k}^{n}}-J_{t_{k-1}^{n}}\right)-\sigma\left(W_{t_{k}^{n}}-W_{t_{k-1}^{n}}\right)$. Ideally, we hope that the estimator of $\mu_{1}$ is $\frac{1}{n h_{n}} \sum_{k=1}^{n}\left(J_{t_{k}^{n}}-J_{t_{k-1}^{n}}\right)$, but we cannot observe a discrete sample $J^{n}=\left\{J_{t_{i}^{n}} t_{i}^{n}=\right.$ $\left.i h_{n} ; i=0,1,2 \ldots, n\right\}$. To this end, we introduce a threshold technique. Motivated by Shimizu [29,30] and Mancini [26,27], we introduce the filter:

$$
\mathcal{D}_{k}^{n}:=\left\{\omega \in \Omega:\left(c h_{n}-Z_{k}\right)>r_{n}\right\},
$$

where $r_{n}>0$ is a suitable threshold parameter dependent on $n$ such that $\lim _{h_{n} \rightarrow 0} r_{n}=0$. Let $\mathcal{C}_{k}^{n}:=\left\{\omega \in \Omega:\left(c h_{n}-Z_{k}\right) \leq r_{n}\right\}$ be the complement of $\mathcal{D}_{k}^{n}$. By (9), if $c h_{n}-Z_{k}>r_{n}$, we can detect the existence of a jump in an interval $\left(t_{k-1}^{n}, t_{k}^{n}\right]$, and then, we take $c h_{n}-Z_{k}$ as an approximation to $J_{t_{k}^{n}}-J_{t_{k-1}^{n}}$. This leads to a natural estimate of $\mu_{1}$ :

$$
\hat{\mu}_{1}=\frac{\sum_{k=1}^{n}\left(c h_{n}-Z_{k}\right) \mathbf{I}_{\mathcal{D}_{k}^{n}}}{n h_{n}} .
$$

Then, $\rho$ is estimated by:

$$
\hat{\rho}=\frac{\sum_{k=1}^{n}\left(c h_{n}-Z_{k}\right) \mathbf{I}_{\mathcal{D}_{k}^{n}}}{c n h_{n}} .
$$

Combining (6), (7), and (11) leads to our estimator:

$$
\hat{\mathcal{F}}_{\Phi}(s)=\frac{i c s(1-\hat{\rho})+\hat{\psi}_{Y}(-s)}{-i s \hat{\psi}_{Y}(-s)} .
$$


Note that the above estimate has no definition at $s=0$. When $s \rightarrow 0, \hat{\psi}_{Y}(-s) \rightarrow 0$, and thus, $\hat{\mathcal{F}}_{\Phi}(s)$ may behave erratically. Applying the inverse Fourier transform and removing a small neighborhood of $s=0$, we propose to estimate $\Phi(u)$ by:

$$
\hat{\Phi}(u)=\frac{1}{2 \pi} \int_{m_{n}}^{M_{n}} e^{-i u s} \hat{\mathcal{F}}_{\Phi}(s) d s+\frac{1}{2 \pi} \int_{-M_{n}}^{-m_{n}} e^{-i u s} \hat{\mathcal{F}}_{\Phi}(s) d s, \quad u>0,
$$

where $m_{n}$ and $M_{n}$ are positive threshold numbers such that $m_{n} \rightarrow 0$ and $M_{n} \rightarrow \infty$ as $n \rightarrow \infty$.

\section{Asymptotic Properties of Estimators}

In this section, the asymptotic properties of $\hat{\rho}$ and $\hat{\Phi}(u)$ are studied. For the ease of exposure, we first introduce some notations. For integer $k=1,2, \mu_{k}:=\int_{0}^{\infty} x^{k} v(d x)$. For any two positive sequences $\left\{x_{n}\right\}_{n=1}^{\infty}$ and $\left\{y_{n}\right\}_{n=1}^{\infty}, x_{n} \lesssim y_{n}$ means that $x_{n} \leq C y_{n}$ for some constant $C$ and large index $n$. For any function $f(x)$ with support $(0, \infty)$, define $\|f\|^{2}=\int_{0}^{\infty} f^{2}(x) d x$. Let $\mu_{L}=\int_{1}^{\infty} x v(d x), \mu_{\gamma}=E\left[\gamma_{k}\right], \sigma_{\gamma}^{2}=\operatorname{Var}\left(\gamma_{k}\right)$, and $\sigma_{M}^{2}=\int_{0}^{1} x^{2} v(d x)$. Next, we make the following assumptions for our theoretical results:

Assumption 1. The safety loading condition holds, i.e., $c-\mu_{1}<\infty$.

Assumption 2. $J_{t}$ is the tempered $\alpha$-stable subordinator.

Assumption 3. $h_{n}=n^{-\kappa_{1}}, m_{n}=O\left(n^{-\kappa_{2}}\right), \alpha \in[0,1)$, and $\theta \in\left(0, \frac{1}{2}\right)$, where $\kappa_{1}, \kappa_{2}>0$, $\kappa_{1}+3 \kappa_{2}<1,1-\kappa_{1}(2-2 \theta)+\kappa_{2}<0$, and $\kappa_{2}-2 \theta \kappa_{1}(1-\alpha)<0$.

Assumption 1 guarantees that the ruin is not a certain event. Assumption 2 means that $v$ has a density of the form $v(x)=\frac{z e^{-\lambda x}}{x^{1+\alpha}} \mathbf{I}_{x>0}$, where $z>0, \lambda>0$, and $\alpha \in[0,1)$. Assumption 2 implies that $\mu_{1}<\infty, \mu_{2}<\infty$,

$$
\begin{gathered}
\int_{0}^{r_{n}} x^{k} v(d x) \sim\left(r_{n}\right)^{k-\alpha}, \quad k=1,2,3,4, \\
\text { and } \int_{r_{n}}^{1} x v(d x) \sim\left(m+r_{n}^{1-\alpha}\right),
\end{gathered}
$$

where $m$ indicates a generic constant.

To establish the convergence rate of $\hat{\Phi}(u)$, we need to calibrate the estimation errors of $\hat{\rho}$. The following Theorem 1 gives the rate of convergence of $\hat{\rho}$.

Theorem 1. Let $r_{n}=h_{n}^{\theta}$ with $\theta \in(0,1 / 2)$. Then, under Assumptions 1 and 2,

$$
\hat{\rho}-\rho=\frac{1}{\sqrt{n h_{n}}} Q_{h_{n}}+O_{P}\left(h_{n}^{\theta(1-\alpha)}+n h_{n}^{2-2 \theta}\right),
$$

where $Q_{h_{n}} \stackrel{D}{\rightarrow} \mathcal{N}\left(0, \frac{1}{c^{2}}\left(\mu_{L} \mu_{\gamma}+\frac{\mu_{L} \sigma_{\gamma}^{2}}{\mu_{\gamma}}+\sigma_{M}^{2}\right)\right)$. 
Proof. Let $\Delta_{k} J=\left(J_{t_{k}^{n}}-J_{t_{k-1}^{n}}\right), \Delta_{k} M=\left(M_{t_{k}^{n}}-M_{t_{k-1}^{n}}\right), \Delta_{k} L=\left(L_{t_{k}^{n}}-L_{t_{k-1}^{n}}\right), \Delta_{k} W=\sigma\left(W_{t_{k}^{n}}-\right.$ $\left.W_{t_{k-1}^{n}}\right)$, and $\Delta_{k} N=\left(N_{t_{k}^{n}}-N_{t_{k-1}^{n}}\right)$. By (9) and (11), we have:

$$
\begin{aligned}
\hat{\rho}-\rho & =\frac{\sum_{k=1}^{n}\left(c h_{n}-Z_{k}\right) \mathbf{I}_{\mathcal{D}_{k}^{n}}}{c n h_{n}}-\frac{1}{c} \mu_{1} \\
& =\frac{1}{c}\left[\frac{\sum_{k=1}^{n}\left(\Delta_{k} J-\Delta_{k} W\right) \mathbf{I}_{\left\{\Delta_{k} J-\Delta_{k} W>h_{n}^{\theta}\right\}}}{n h_{n}}-\mu_{1}\right] \\
& =\frac{1}{c}\left[\frac{\sum_{k=1}^{n}\left(b h_{n}+\Delta_{k} L-\Delta_{k} W+\Delta_{k} M\right) \mathbf{I}_{\left\{b h_{n}+\Delta_{k} L-\Delta_{k} W+\Delta_{k} M>h_{n}^{\theta}\right\}}}{n h_{n}}-\mu_{1}\right] \\
& =\sum_{j=1}^{4} I_{j},
\end{aligned}
$$

where:

$$
\begin{gathered}
I_{1}=\frac{1}{c}\left[\frac{\sum_{k=1}^{n}\left(\Delta_{k} L-\Delta_{k} W\right) \mathbf{I}_{\left\{\Delta_{k} L-\Delta_{k} W>h_{n}^{\theta}\right\}}}{n h_{n}}-\int_{1}^{\infty} x v(d x)\right], \\
I_{2}=\frac{1}{c}\left[\frac{\sum_{k=1}^{n}\left(\Delta_{k} L-\Delta_{k} W\right)}{n h_{n}}\left(\mathbf{I}_{\left\{b h_{n}+\Delta_{k} L-\Delta_{k} W+\Delta_{k} M>h_{n}^{\theta}\right\}}-\mathbf{I}_{\left\{\Delta_{k} L-\Delta_{k} W>h_{n}^{\theta}\right\}}\right)\right], \\
I_{3}=\frac{1}{c}\left[\frac{\sum_{k=1}^{n}\left(b h_{n}+\Delta_{k} M\right)}{n h_{n}}-\int_{0}^{1} x v(d x)\right], \\
I_{4}=-\frac{1}{c} \frac{\sum_{k=1}^{n}\left(b h_{n}+\Delta_{k} M\right)}{n h_{n}} \mathbf{I}_{\left\{b h_{n}+\Delta_{k} L-\Delta_{k} W+\Delta_{k} M \leq h_{n}^{\theta}\right\}} .
\end{gathered}
$$

In the following, we study each of $I_{1}$ to $I_{4}$.

(i) Note that:

$$
\begin{aligned}
I_{1}= & \frac{1}{c}\left[\frac{\sum_{k=1}^{n}\left(\Delta_{k} L-\Delta_{k} W\right) \mathbf{I}_{\left\{\Delta_{k} L-\Delta_{k} W>h_{n}^{\theta}, \Delta_{k} N=1\right\}}}{n h_{n}}-\mu_{L}\right] \\
& +\frac{1}{c}\left[\frac{\sum_{k=1}^{n}\left(\Delta_{k} L-\Delta_{k} W\right) \mathbf{I}_{\left\{\Delta_{k} L-\Delta_{k} W>h_{n}, \Delta_{k} N=0\right\}}}{n h_{n}}\right] \\
& +\frac{1}{c}\left[\frac{\sum_{k=1}^{n}\left(\Delta_{k} L-\Delta_{k} W\right) \mathbf{I}_{\left\{\Delta_{k} L-\Delta_{k} W>h_{n}^{\theta}, \Delta_{k} N \geq 2\right\}}}{n h_{n}}\right] \\
\equiv & I_{11}+I_{12}+I_{13} .
\end{aligned}
$$

By Mancini [25], $\left\{\Delta_{k} L-\Delta_{k} W>h_{n}^{\theta}\right\}$ is equal to $\left\{\Delta_{k} N=1\right\}$ almost surely for small $h_{n}$. Thus, for small $h_{n}$,

$$
\begin{aligned}
I_{11} & =\frac{1}{c}\left[\frac{\sum_{k=1}^{n}\left(\Delta_{k} L-\Delta_{k} W\right) \mathbf{I}_{\left\{\Delta_{k} L-\Delta_{k} W>h_{n}^{\theta}, \Delta_{k} N=1\right\}}}{n h_{n}}-\mu_{L}\right] \\
& =\frac{1}{c}\left[\frac{\sum_{k=1}^{n}\left(\gamma_{k}-\Delta_{k} W\right) \mathbf{I}_{\left\{\Delta_{k} N=1\right\}}}{n h_{n}}-\mu_{L}\right] .
\end{aligned}
$$

Rewrite:

$$
I_{11}=\frac{1}{\sqrt{n h_{n}}}\left[\frac{\sum_{k=1}^{n}\left\{\left(\gamma_{k}-\Delta_{k} W\right) \mathbf{I}_{\left\{\Delta_{k} N=1\right\}}-h_{n} \mu_{L}\right\}}{c \sqrt{n h_{n}}}\right] \equiv \frac{Z_{h_{n}}}{\sqrt{n h_{n}}} .
$$

Then, due to the independence of $\gamma_{k}, N_{t}$, and $W_{t}$, by the central limit theorem, we have $Z_{h_{n}} \stackrel{D}{\rightarrow} \mathcal{N}\left(0, \frac{1}{c^{2}}\left(\mu_{L} \mu_{\gamma}+\frac{\mu_{L} \sigma_{\gamma}^{2}}{\mu_{\gamma}}\right)\right)$.

By (A.2)-(A.4) in You and Yin [32], we have:

$$
I_{12}=\frac{1}{c}\left[\frac{\sum_{k=1}^{n}\left(\Delta_{k} L-\Delta_{k} W\right) \mathbf{I}_{\left\{\Delta_{k} L-\Delta_{k} W>h_{n}^{\theta}, \Delta_{k} N=0\right\}}}{n h_{n}}\right]=o_{P}\left(\frac{1}{\sqrt{n h_{n}}}\right)
$$


and:

$$
I_{13}=\frac{1}{c}\left[\frac{\sum_{k=1}^{n}\left(\Delta_{k} L-\Delta_{k} W\right) \mathbf{I}_{\left\{\Delta_{k} L-\Delta_{k} W>h_{n}^{\theta}, \Delta_{k} N \geq 2\right\}}}{n h_{n}}\right]=o_{P}\left(\frac{1}{\sqrt{n h_{n}}}\right) .
$$

Therefore, $I_{1}=\frac{Z_{h_{n}}}{\sqrt{n h_{n}}}+o_{P}\left(\frac{1}{\sqrt{n h_{n}}}\right)$.

(ii) Next, we show that $I_{2}=o_{P}\left(h_{n}^{1-\alpha \theta} \sqrt{\log \left(\frac{1}{h_{n}}\right)}\right)$. Since $b h_{n}+\Delta_{k} M \geq 0$, we have $\left\{\Delta_{k} L-\Delta_{k} W>h_{n}^{\theta}\right\} \subseteq\left\{b h_{n}+\Delta_{k} M+\Delta_{k} L-\Delta_{k} W>h_{n}^{\theta}\right\}$. Thus,

$$
\begin{aligned}
I_{2} & =\frac{1}{c}\left[\frac{\sum_{k=1}^{n}\left(\Delta_{k} L-\Delta_{k} W\right)}{n h_{n}}\left(\mathbf{I}_{\left\{b h_{n}+\Delta_{k} L-\Delta_{k} W+\Delta_{k} M>h_{n}^{\theta}\right\}}-\mathbf{I}_{\left\{\Delta_{k} L-\Delta_{k} W>h_{n}^{\theta}\right\}}\right)\right] \\
& =\frac{1}{c}\left[\frac{\sum_{k=1}^{n}\left(\Delta_{k} L-\Delta_{k} W\right)}{n h_{n}} \mathbf{I}_{\left\{b h_{n}+\Delta_{k} L-\Delta_{k} W+\Delta_{k} M>h_{n}^{\theta}, \Delta_{k} L-\Delta_{k} W \leq h_{n}^{\theta}\right\}}\right] .
\end{aligned}
$$

For small $h_{n},\left\{\Delta_{k} L-\Delta_{k} W \leq h_{n}^{\theta}\right\}=\left\{\Delta_{k} N=0\right\}$ almost surely. As a result, we have:

$$
I_{2}=\frac{1}{c}\left[\frac{\sum_{k=1}^{n}\left(-\Delta_{k} W\right)}{n h_{n}} \mathbf{I}_{\left\{\Delta_{k} M>h_{n}^{\theta}, \Delta_{k} L-\Delta_{k} W \leq h_{n}^{\theta}\right\}}\right]\left(1+o_{p}(1)\right) .
$$

By Mancini [27], we obtain that:

$$
\frac{1}{c}\left[\frac{\sum_{k=1}^{n}\left|\Delta_{k} W\right|}{n h_{n}} \mathbf{I}_{\left\{\Delta_{k} M>h_{n}^{\theta}\right\}}\right]=o_{P}\left(h_{n}^{1-\alpha \theta} \sqrt{\log \left(\frac{1}{h_{n}}\right)}\right) .
$$

(iii) Applying the central limit theorem, we obtain:

$$
I_{3}=\frac{1}{c}\left[\frac{\sum_{k=1}^{n}\left(b h_{n}+\Delta_{k} M\right)}{n h_{n}}-\int_{0}^{1} x v(d x)\right] \equiv \frac{T_{h_{n}}}{\sqrt{n h_{n}}},
$$

where $T_{h_{n}} \stackrel{D}{\rightarrow} \mathcal{N}\left(0, \frac{\sigma_{M}^{2}}{c^{2}}\right)$.

(iv) Now, let us consider the last term of (17).

$$
\begin{aligned}
-I_{4}= & \frac{1}{c} \frac{\sum_{k=1}^{n}\left(b h_{n}+\Delta_{k} M\right)}{n h_{n}} \mathbf{I}_{\left\{b h_{n}+\Delta_{k} L-\Delta_{k} W+\Delta_{k} M \leq h_{n}^{\theta}\right\}} \\
= & \frac{1}{c} \frac{\sum_{k=1}^{n}\left(b h_{n}+\Delta_{k} M\right)}{n h_{n}} \mathbf{I}_{\left\{b h_{n}+\Delta_{k} L-\Delta_{k} W+\Delta_{k} M \leq h_{n}^{\theta}, b h_{n}+\Delta_{k} M>2 h_{n}^{\theta}\right\}} \\
& +\frac{1}{c} \frac{\sum_{k=1}^{n}\left(b h_{n}+\Delta_{k} M\right)}{n h_{n}} \mathbf{I}_{\left\{b h_{n}+\Delta_{k} L-\Delta_{k} W+\Delta_{k} M \leq h_{n}^{\theta}, b h_{n}+\Delta_{k} M \leq 2 h_{n}^{\theta}\right\}} \\
\equiv & B_{1}+B_{2} .
\end{aligned}
$$

By (A.29)-(A.32) in You and Yin [32], we have

$$
B_{1}=\frac{1}{c} \frac{\sum_{k=1}^{n}\left(b h_{n}+\Delta_{k} M\right)}{n h_{n}} \mathbf{I}_{\left\{b h_{n}+\Delta_{k} L-\Delta_{k} W+\Delta_{k} M \leq h_{n}^{\theta}, b h_{n}+\Delta_{k} M>2 h_{n}^{\theta}\right\}} \leq O_{p}\left(n h_{n}^{1+(1-2 \theta)}\right) .
$$


Next, we show that the second term of (23) is of order $O\left(r_{n}^{1-\alpha}\right)$. In fact,

$$
\begin{aligned}
B_{2} & =\frac{1}{c} \frac{\sum_{k=1}^{n}\left(b h_{n}+\Delta_{k} M\right)}{n h_{n}} \mathbf{I}_{\left\{b h_{n}+\Delta_{k} L-\Delta_{k} W+\Delta_{k} M \leq h_{n}^{\theta}, b h_{n}+\Delta_{k} M \leq 2 h_{n}^{\theta}\right\}} \\
& \leq \frac{1}{c} \frac{\sum_{k=1}^{n}\left(b h_{n}+\Delta_{k} M\right)}{n h_{n}} \mathbf{I}_{\left\{b h_{n}+\Delta_{k} M \leq 2 h_{n}^{\theta}\right\}} \\
& =\frac{1}{c} \frac{\sum_{k=1}^{n}\left(\int_{t_{k-1}^{n}}^{t_{k}^{n}} \int_{0}^{2 h_{n}^{\theta}} x \mu(d x, d t)\right)}{n h_{n}} \mathbf{I}_{\left\{b h_{n}+\Delta_{k} M \leq 2 h_{n}^{\theta}\right\}} \\
& \leq \frac{1}{c} \frac{\sum_{k=1}^{n}\left(\int_{t_{k-1}^{n}}^{t_{k}^{n}} \int_{0}^{2 h_{n}^{\theta}} x \mu(d x, d t)\right)}{n h_{n}} .
\end{aligned}
$$

Using Assumption 2 and the law of large numbers, we establish that:

$$
E\left[\frac{1}{c} \frac{\sum_{k=1}^{n}\left(\int_{t_{k-1}^{n}}^{t_{k}^{n}} \int_{0}^{2 h_{n}^{\theta}} x \mu(d x, d t)\right)}{n h_{n}}\right]=\frac{1}{c} \int_{0}^{2 h_{n}^{\theta}} x v(d x) \sim h_{n}^{\theta(1-\alpha)}
$$

and:

$$
B_{2}=O\left(h_{n}^{\theta(1-\alpha)}\right)
$$

Thus, $I_{4}=O_{p}\left(n h_{n}^{1+(1-2 \theta)}+h_{n}^{\theta(1-\alpha)}\right)$.

Finally, combining (i)-(iv) leads to $\hat{\rho}-\rho=\frac{1}{\sqrt{n h_{n}}}\left(Z_{h_{n}}+T_{h_{n}}\right)+O_{p}\left(n h_{n}^{1+(1-2 \theta)}+\right.$ $\left.h_{n}^{\theta(1-\alpha)}\right)$. Note that $Z_{h_{n}}$ and $T_{h_{n}}$ are independent. It follows that the result of the theorem holds.

The convergence rate of $\hat{\Phi}(u)$ depends on the choice of $h_{n}, m_{n}$, and $M_{n}$. The following theorem establishes the convergence rate of the integrated squared error of $\hat{\Phi}(u)$.

Theorem 2. Under Assumptions 1-3, as $n \rightarrow \infty$,

$$
\|\hat{\Phi}(u)-\Phi(u)\|^{2}=O_{P}\left(\max \left\{n^{\kappa_{1}+3 \kappa_{2}-1}, n^{\kappa_{2}-2 \kappa_{1} \theta(1-\alpha)}, n^{1-\kappa_{1}(2-2 \theta)+\kappa_{2}}, \frac{1}{M_{n}}\right\}\right) .
$$

Proof. By (13), we have:

$$
\|\hat{\Phi}(u)-\Phi(u)\|^{2}=\int_{0}^{\infty}\left|\frac{1}{2 \pi} \int_{m_{n}}^{M_{n}} e^{-i u s} \hat{\mathcal{F}}_{\Phi}(s) d s+\frac{1}{2 \pi} \int_{-M_{n}}^{-m_{n}} e^{-i u s} \hat{\mathcal{F}}_{\Phi}(s) d s-\Phi(u)\right|^{2} d u .
$$

Using Parseval's identity, we obtain that:

$$
\begin{aligned}
\|\hat{\Phi}(u)-\Phi(u)\|^{2} \lesssim & \frac{1}{2 \pi} \int_{m_{n}<|s|<M_{n}}\left|\frac{c(1-\hat{\rho})}{\hat{\psi}_{Y}(-s)}-\frac{c(1-\rho)}{\psi_{Y}(-s)}\right|^{2} d s \\
& +\frac{1}{2 \pi} \int_{|s| \geq M_{n}}\left|\mathcal{F}_{\Phi}(s)\right|^{2} d s+\frac{1}{2 \pi} \int_{|s| \leq m_{n}}\left|\mathcal{F}_{\Phi}(s)\right|^{2} d s \\
= & \amalg_{1}+\amalg_{2}+\amalg_{3} .
\end{aligned}
$$


Note that:

$$
\begin{aligned}
\amalg_{1}= & \frac{1}{2 \pi} \int_{m_{n}<|s|<M_{n}}\left|\frac{\left(c-\mu_{1}\right)\left[\hat{\psi}_{Y}(-s)-\psi_{Y}(-s)\right]+c \psi_{Y}(-s)[\hat{\rho}-\rho]}{\hat{\psi}_{Y}(-s) \psi_{Y}(-s)}\right|^{2} d s \\
\lesssim & \int_{m_{n}<|s|<M_{n}}\left|\frac{\left(c-\mu_{1}\right)\left[\hat{\psi}_{Y}(-s)-\psi_{Y}(-s)\right]}{\hat{\psi}_{Y}(-s) \psi_{Y}(-s)}\right|^{2} d s \\
& +\int_{m_{n}<|s|<M_{n}}\left|\frac{c[\hat{\rho}-\rho]}{\hat{\psi}_{Y}(-s)}\right|^{2} d s .
\end{aligned}
$$

Using the fact that $\left|\psi_{Y}(-s)\right|>s\left(c-\mu_{1}\right)$ and Theorem 4.1 in You and Cai [33], we establish that:

$$
\int_{m_{n}<|s|<M_{n}}\left|\frac{\left(c-\mu_{1}\right)\left[\hat{\psi}_{Y}(-s)-\psi_{Y}(-s)\right]}{\hat{\psi}_{Y}(-s) \psi_{Y}(-s)}\right|^{2} d s=O_{P}\left(\frac{1}{n h_{n}} m_{n}^{-3}\right)
$$

and:

$$
\int_{m_{n}<|s|<M_{n}}\left|\frac{c[\hat{\rho}-\rho]}{\hat{\psi}_{Y}(-s)}\right|^{2} d s=O_{P}\left(\frac{1}{n h_{n}} \frac{1}{m_{n}}\right)+O_{P}\left(\left(n h_{n}^{2-2 \theta}+h_{n}^{2 \theta(1-\alpha)}\right) \frac{1}{m_{n}}\right) .
$$

By Lemma 1 in Zhang and Yang [11], we have $\amalg_{2}=O\left(\frac{1}{M_{n}}\right)$ and $\amalg_{3}=O\left(m_{n}\right)$. Then, combining (30), (31), and Assumption 3 leads to:

$$
\|\hat{\Phi}(u)-\Phi(u)\|^{2}=O_{P}\left(\max \left\{n^{\kappa_{1}+3 \kappa_{2}-1}, n^{\kappa_{2}-2 \kappa_{1} \theta(1-\alpha)}, n^{1-\kappa_{1}(2-2 \theta)+\kappa_{2}}, \frac{1}{M_{n}}\right\}\right) .
$$

\section{Conclusions}

In this paper, the threshold and Fourier transform (inversion) techniques were employed to construct a new estimator of ruin probability for the spectrally negative Lévy process. The convergence rate of the integrated squared error (ISE) of the estimator was obtained when the jump component was the tempered $\alpha$-stable subordinator. This shows that the ISE of the estimated ruin probability function is well controlled. Further work includes, but is not limited to deriving the asymptotic distribution of the proposed estimator and making statistical inference for ruin probability, under the framework that the risk model is a spectrally negative Lévy process with dividend strategy and investment. Furthermore, statistical inference for the Gerber-Shiu function and the dividend function are worthy of study.

Author Contributions: Formal analysis, H.Y.; Methodology, H.Y.; Writing, Y.G. All authors have read and agreed to the published version of the manuscript.

Funding: This research was funded by Natural Science Foundation of Shandong Province (No. ZR2020QA029).

Informed Consent Statement: Not applicable.

Acknowledgments: The authors express many thanks to the anonymous referees for their helpful comments.

Conflicts of Interest: The authors declare no conflict of interest.

\section{References}

1. Croux, K.; Veraverbeke, N. Non-parametric estimators for the probability of ruin. Insur. Math. Econ. 1990, 9, 127-130. [CrossRef]

2. Frees, E.W. Nonparametric estimation of the probability of ruin. Astin Bull. 1986, 16, 81-90. [CrossRef] 
3. Mnatsakanov, R.; Ruymgaart, L.L.; Ruymgaart, F.H. Nonparametric estimation of ruin probabilities given a random sample of claims. Math. Methods Stat. 2008, 17, 35-43. [CrossRef]

4. Pitts, S.M. Nonparametric estimation of compound distributions with applications in insurance. Ann. Inst. Stat. Math. 1994, 46, 537-555.

5. Politis, K. Semiparametric estimation for non-ruin probabilities. Scand. Actuar. J. 2003, 2003, 75-96. [CrossRef]

6. Veraverbeke, N. Asymptotic estimates for the probability of ruin in a Poisson model with diffusion. Insur. Math. Econ. 1993, 13, 57-62. [CrossRef]

7. Huang, Y.; Li, J.; Liu, H.; Yu, W. Estimating ruin probability in an insurance risk model with stochastic premium income based on the CFS method. Mathematics 2021, 9, 982. [CrossRef]

8. Li, J.; Yu, W.; Liu, C. Nonparametric estimation of ruin probability by complex Fourier series expansion in the compound Poisson mode. Commun. Stat.-Theory Methods 2020. [CrossRef]

9. You, H.; Guo, J.; Jiang, J. Interval estimation of the ruin probability in the classical compound Poisson risk model. Comput. Stat. Data Anal. 2020, 144, 106890. [CrossRef]

10. Zhang, Z.; Yang, H. Nonparametric estimate of the ruin probability in a pure-jump Lévy risk model. Insur. Math. Econ. 2013, 53, 24-35. [CrossRef]

11. Zhang, Z.; Yang, H. Nonparametric estimation for the ruin probability in a Lévy risk model under low-frequency observation. Insur. Math. Econ. 2014, 59, 168-177. [CrossRef]

12. Zhang, Z. Nonparametric estimation of the finite time ruin probability in the classical risk model. Scand. Actuar. J. 2017, 5, 452-469. [CrossRef]

13. Zhang, Z.; Yang, H.; Yang, H. On a nonparametric estimator for ruin probability in the classical risk model. Scand. Actuar. J. 2014, 4, 309-338. [CrossRef]

14. Su, W.; Yu, W. Asymptotically normal estimators of the Gerber-Shiu function in classical insurance risk model. Mathematics 2020, 8, 1638. [CrossRef]

15. Su, W.; Yu, W. Estimating the Gerber-Shiu Function in Lévy Insurance Risk Model by Fourier-Cosine Series Expansion. Mathematics 2021, 9, 1402. [CrossRef]

16. Yang, Y.; Su, W.; Zhang, Z. Estimating the discounted density of the deficit at ruin by Fourier cosine series expansion. Stat. Probab. Lett. 2019, 146, 147-155. [CrossRef]

17. Zhang, Z.; Su, W. Estimating the Gerber-Shiu function in a Lévy risk model by Laguerre series expansion. J. Comput. Appl. Math. 2019, 346, 133-149. [CrossRef]

18. Su, W.; Yong, Y.; Zhang, Z. Estimating the Gerber-Shiu function in the perturbed compound Poisson model by Laguerre series expansion. J. Math. Anal. Appl. 2019, 469, 705-729. [CrossRef]

19. Zhang, Z.; Su, W. A new efficient method for estimating the Gerber-Shiu function in the classical risk model. Scand. Actuar. J. 2018, 5, 426-449. [CrossRef]

20. Zhang, Z. Estimating the Gerber-Shiu function by Fourier-Sinc series expansion. Scand. Actuar. J. 2017, 10, 898-919. [CrossRef]

21. Shimizu, Y. Non-parametric estimation of the Gerber-Shiu function for the Wiener-Poisson risk model. Scand. Actuar. J. 2012, 2012, 56-69. [CrossRef]

22. Shimizu, Y.; Zhang, Z. Estimating Gerber-Shiu functions from discretely observed Lévy driven surplus. Insur. Math. Econ. 2017, 74, 84-98. [CrossRef]

23. Asmussen, S.; Albrecher, H. Ruin Probabilities, 2nd ed.; World Scientific: Singapore, 2010.

24. Comte, F.; Genon-Catalot, V. Nonparamtetric estimation for pure jump Lévy processes based on high frequency data. Stoch. Process. Their Appl. 2009, 119, 4088-4123. [CrossRef]

25. Mancini, C. Estimation of the characteristics of the jump of a general Poisson-diffusion model. Scand. Actuar. J. 2004, 1, 42-52. [CrossRef]

26. Mancini, C. Non-parametric threshold estimation for models with stochastic diffusion coefficient and jumps. Scand. J. Stat. 2009, 36, 270-296. [CrossRef]

27. Mancini, C. Non-parametric tests for pathwise properties of semimartingales. Bernoulli 2011, 17, 781-813.

28. Mancini, C. The speed of convergence of the threshold estimator of integrated variance. Stoch. Process. Their Appl. 2011, 121, 845-855. [CrossRef]

29. Shimizu, Y. A new aspect of a risk process and its statistical inference. Insur. Math. Econ. 2009, 44, 70-77. [CrossRef]

30. Shimizu, Y. Functional estimation for Lévy measures of semimartingales with Poissonian jumps. J. Multivar. Anal. 2009, 100, 1073-1092. [CrossRef]

31. Sato, K. Lévy Processes and Infinitely Divisible Distributions; Cambridge University Press: Cambridge, UK, 1999.

32. You, H.; Yin, C. Threshold estimation for a spectrally negative Lévy process. Math. Probl. Eng. 2020, 1, 1-12. [CrossRef]

33. You, H.; Cai, C. Nonparametric estimation for a spectrally negative Lévy process based on high frequency data. J. Comput. Appl. Math. 2019, 345, 196-205. [CrossRef] 